\title{
COPD and osteoporosis: links, risks, and treatment challenges
}

\author{
This article was published in the following Dove Press journal: \\ International Journal of COPD \\ 29 March 2016 \\ Number of times this article has been viewed
}

\section{Daisuke Inoue \\ Reiko Watanabe \\ Ryo Okazaki}

Division of Endocrinology and Metabolism, Third Department of Medicine, Teikyo University Chiba Medical Center, Ichihara, Chiba, Japan

\begin{abstract}
Chronic obstructive pulmonary disease (COPD) is a chronic inflammatory airway disease associated with various systemic comorbidities including osteoporosis. Osteoporosis and its related fractures are common and have significant impacts on quality of life and even respiratory function in patients with COPD. COPD-associated osteoporosis is however extremely undertreated. Recent studies have suggested that both decreased bone mineral density (BMD) and impaired bone quality contribute to bone fragility, causing fractures in COPD patients. Various clinical risk factors of osteoporosis in COPD patients, including older age, emaciation, physical inactivity, and vitamin D deficiency, have also been described. It is critically important for pulmonologists to be aware of the high prevalence of osteoporosis in COPD patients and evaluate them for such fracture risks. Routine screening for osteoporosis will enable physicians to diagnose COPD patients with comorbid osteoporosis at an early stage and give them appropriate treatment to prevent fracture, which may lead to improved quality of life as well as better long-term prognosis.
\end{abstract}

Keywords: fracture, bone mineral density, bone quality, bone turnover, vitamin D

\section{Introduction}

Chronic obstructive pulmonary disease (COPD) is defined as a common preventable and treatable disease characterized by persistent airflow limitation that is usually progressive and associated with an enhanced chronic inflammatory response in the airways and the lung to noxious particles or gases, particularly inhaled cigarette smoke. COPD is now recognized as a systemic disease complicated with various comorbidities including lung cancer, atherosclerosis, muscle wasting, osteoporosis, diabetes, and anxiety/depression. ${ }^{1-3}$ Management of these comorbidities is clinically important as they are associated with hospitalization, mortality, and diminished quality of life in COPD subjects. ${ }^{3-5}$

Osteoporosis is among the major systemic comorbidities of COPD. Although the causal relationship and molecular link between COPD and osteoporosis remain to be established, recent epidemiological data clearly indicate that osteoporosis is highly prevalent in COPD patients. ${ }^{6-11}$ A retrospective chart review on 234 male subjects referred for osteoporosis in a single bone clinic revealed that COPD was the leading cause of secondary osteoporosis at the time of referral. ${ }^{12}$ Moreover, osteoporosis-associated fractures may further deteriorate pulmonary function and impair activities of daily life of COPD patients. Thus, the two diseases will form a vicious cycle, causing significant burden on these patients. Osteoporosis in COPD patients is however extremely undertreated., ${ }^{73-15}$ In this narrative review, we will summarize clinical links and the mutual relationship between COPD and osteoporosis, 
as well as pathophysiology of osteoporosis in COPD patients, touching on the issue of bone quality and metabolism, and we will finally discuss diagnosis and management of COPDassociated osteoporosis.

\section{Clinical links between COPD and osteoporosis \\ Osteoporosis, bone mineral density, and bone quality}

Osteoporosis is defined as a skeletal disorder characterized by compromised bone strength, predisposing a person to an increased risk of fracture. The fracture risk depends on bone strength, which is determined by bone mineral density (BMD) and bone quality. ${ }^{16}$ However, due to the absence of clinical tools for precise evaluation of bone quality, a diagnosis of osteoporosis has been reliant on BMD measured by dual-energy X-ray absorptiometry (DXA): osteoporosis is diagnosed when $\mathrm{BMD}$ is 2.5 standard deviations or more below the young adult mean ( $T$-score is equal to or less than -2.5 ) according to the World Health Organization criteria. ${ }^{17}$ Thus, previous studies reporting prevalence of "osteoporosis" in COPD should be interpreted with caution, as most of them referred to low BMD as osteoporosis, while some reports specifically analyzed fracture prevalence.

It is generally accepted that BMD accounts for approximately $70 \%$ of bone strength. ${ }^{16}$ By definition, any determinants of bone strength other than BMD are referred to as "bone quality", which contributes to the rest of bone strength. Bone mostly consists of type 1 collagen and hydroxyapatite crystals, and bone quality depends on the material properties of these constituents as well as the three-dimensional microarchitecture of the bone. In some forms of secondary osteoporosis, such as those associated with diabetes and glucocorticoid (GC) excess, fracture risk depends less on $\mathrm{BMD}$ and more on bone quality when compared to primary osteoporosis, even though mechanisms of deteriorated bone quality are incompletely understood. ${ }^{18-20}$ Whether or not COPD-associated fracture risk involves impaired bone quality is not only a matter of great interest but may also be a critical issue influencing therapeutic strategy.

\section{Osteoporotic fractures}

Primary osteoporosis can occur in both sexes at all ages, but is 3-4 times more common in women than men. Age is one of the strongest risk factors, particularly for vertebral and hip fractures. Morphometric vertebral fractures identified by X-ray exams are the most common form of fracture and can readily occur from early 60s. A prevalence rate of vertebral fracture in women above 50 years of age is as high as $25 \%$ or more. Vertebral fractures are often asymptomatic, and only one-third are recognized as clinical fractures with symptoms. ${ }^{21,22}$ Furthermore, as discussed earlier, fractures can occur even with BMD above the threshold of osteoporosis, ie, $T$-score $>-2.5$, because of deteriorated bone quality. ${ }^{23}$ It is thus important to assess prevalent morphometric fractures by spinal X-rays. The severity of vertebral fractures can be morphologically assessed according to the semiquantitative method of Genant et al. ${ }^{24}$ Hip fractures are usually a problem of women in their late 70 s and 80 s, and have a profound impact on mortality and quality of life. Nonhip, nonvertebral fractures such as wrist fractures can occur throughout all ages, beginning from 50s. ${ }^{16,21,25}$

\section{Low BMD in COPD}

In a previous systematic review including a total of $775 \mathrm{COPD}$ patients from 13 studies, ${ }^{26-38}$ the overall prevalence of osteoporosis defined by low BMD was $35.1 \%$ on average, ranging from $8.7 \%$ to $69 \%{ }^{6}$ The papers reporting the three highest numbers, however, involved either seriously ill patients awaiting lung transplantation $\left(59 \%^{27}\right.$ and $\left.69 \%{ }^{38}\right)$ or patients who were hospitalized due to acute exacerbation $\left(60 \%{ }^{35}\right)$, whereas the study that reported the lowest number $\left(8.7 \%{ }^{31}\right)$ used calcaneal ultrasonography instead of DXA for BMD evaluation. More recent studies recruiting stable outpatients demonstrated that prevalence of low BMD ( $T$-score $\leq-2.5)$ was approximately $22 \%-42 \%$ (Table 1 )..$^{7,10,39-43}$

The absolute prevalence is however difficult to interpret without comparison to control subjects. The National Health and Nutrition Examination Survey (NHANES) demonstrated a $16.9 \%$ prevalence of osteoporosis in 995 COPD subjects and an $8.9 \%$ prevalence in 14,828 non-COPD subjects aged 45 years or older. ${ }^{41}$ Thus, COPD significantly increased the risk of osteoporosis (low BMD) 1.9-fold, although COPD subjects were 2.7 years older and included $7.1 \%$ more females in this study. Most other cross-sectional studies comparing COPD patients with controls and population-based cohort studies have also estimated the COPD-associated risk of osteoporosis at approximately 1.5-2-fold. 26,30,44,45

\section{Prevalent vertebral fractures in COPD}

As summarized in Table 1, prevalence of morphometric vertebral fractures in COPD patients has been reported to be 24\%-79\%. ${ }^{7,10,14,28,46-50}$ Again, the values vary widely depending on the characteristics of study subjects such as age, sex, and COPD severity. Some studies included controls for 
comparison. One study reported that a significantly greater proportion of COPD patients had at least one severe vertebral fracture, with odds ratio (OR) being 3.75 compared with ageand sex-matched controls. ${ }^{47}$ Another study demonstrated that the prevalence of vertebral fractures was $72 \%$ higher in 465 COPD subjects than in 462 controls. $^{49}$

Interestingly, in the latter study, COPD patients appeared to have more thoracic fractures than lumbar fractures, although both COPD and control subjects had fractures most frequently in the midthoracic region (Th7-8) and the thoracic-lumbar junction (Th12-L1). ${ }^{49}$ Others reported similar results, that $49 \%$ of the subjects had at least one thoracic fracture, whereas $16.5 \%$ had at least one lumbar fracture. ${ }^{46}$ Another study of 255 COPD outpatients reported 139 thoracic and 33 lumbar vertebral fractures. ${ }^{10}$ One could only speculate that thoracic vertebrae may preferentially be affected by aberrant physical/mechanical interaction between the lungs and the thoracic cage in the context of thoracic deformities, altered lung compliance, frequent coughing, and respiratory muscle wasting as well as biochemical interaction through putative soluble factors in the local milieu. Predominance of thoracic fractures is still a matter of controversy and requires further investigation.

\section{Clinical fractures in COPD}

Clinical fractures are those with pain and other symptoms, usually bringing a patient to clinical attention. In a large observational cohort study called Global Longitudinal Study of Osteoporosis Women (GLOW), 6.2\% of 52,960 subjects sustained an incident clinical fracture over 2 years. ${ }^{51}$ COPD was among the comorbidities associated with significantly increased fracture incidence (age-adjusted hazard ratio $=1.5$, 95\% confidence interval [CI]: 1.3-1.7), but no information about the fracture site was provided. ${ }^{51}$ A large case-control study (108,754 cases) using UK general practice research database reported that crude OR $(95 \% \mathrm{CI})$ for osteoporotic fractures was $1.61(1.52-1.71)$ in COPD/asthma patients when compared with controls. ${ }^{52}$ The authors also reported significantly increased incidence of hip and clinical vertebral fractures in COPD. A similar report from Denmark with 124,655 cases also demonstrated that any osteoporotic fractures were increased, with crude OR $(95 \% \mathrm{CI})$ of 1.89 $(1.81-1.96) .{ }^{53}$

A more recent cohort study in UK estimated fracture risk in 3,142,673 primary care patients with various comorbidities and reported that adjusted hazard ratio $(95 \% \mathrm{CI})$ of COPD for hip fracture was 1.23 (1.16-1.31) in women and 1.34 (1.22-1.48) in men. ${ }^{54}$ Absolute hip fracture rate in a retroprospective cohort study of 87,360 men aged 50 and older (66.8 on the average) diagnosed with COPD in USA was reported to be 3.99 per 1,000 person-years, which was several times higher than that in the general population. ${ }^{55}$ Collectively, COPD does appear to increase the risk of clinical osteoporotic fractures, including hip fracture.

Regan et $\mathrm{al}^{56}$ investigated the impact of COPD on hip fracture outcomes in a large cohort of men $(n=12,646)$ undergoing hip fracture surgery. Surprisingly, COPD was noted in $47 \%$ of the hip fracture cases, and $26 \%$ had severe COPD and showed a significantly higher 1-year mortality of $40.2 \%$ compared with $31.0 \%$ in mild COPD and $28.8 \%$ in non-COPD subjects. Thus, although the true incidence of hip fracture in COPD still remains to be determined, the presence of COPD in patients with hip fractures is common and is associated with a poor prognosis.

\section{Pathophysiology of COPD- associated osteoporosis Bone quality in COPD}

Previous studies investigating both BMD and vertebral fracture prevalence in COPD subjects suggested that BMD was not a good predictor of fracture. In a study by Graat-Verboom et al, ${ }^{10} 36.5 \%$ of the patients had vertebral fractures, while $23.6 \%$ showed BMD-defined osteoporosis $(T \leq 2.5)$. A Japanese study of 136 COPD males reported prevalent vertebral fractures in $79.4 \%$ and low BMD $(T \leq 2.5)$ only in $38.8 \%$ patients. ${ }^{7}$ These results support BMD-independent mechanisms in COPD-associated osteoporosis.

Data on bone quality in COPD patients is however limited. Particularly, there have been no reports on material properties of bone in COPD. As for structural properties, bone biopsy is by far the best way to directly assess bone microarchitecture at the tissue level. Histomorphometric analysis of biopsied bone specimens from postmenopausal women with COPD who had not been treated with systemic GCs has been reported. ${ }^{57}$ COPD women showed significantly decreased trabecular bone volume and connective density as well as decreased cortical width and increased cortical porosity, compared with age-matched postmortem control samples. ${ }^{57}$ Connectivity density was found to be negatively correlated with smoking (pack-years) in this study. ${ }^{57}$

The trabecular bone score (TBS) is a gray-level textural metric that can be extracted from the two-dimensional lumbar spine DXA image and reflects the microarchitecture of trabecular bone. ${ }^{58}$ TBS is only partially dependent on $\mathrm{BMD}$, and lower TBS has been shown to be associated with 


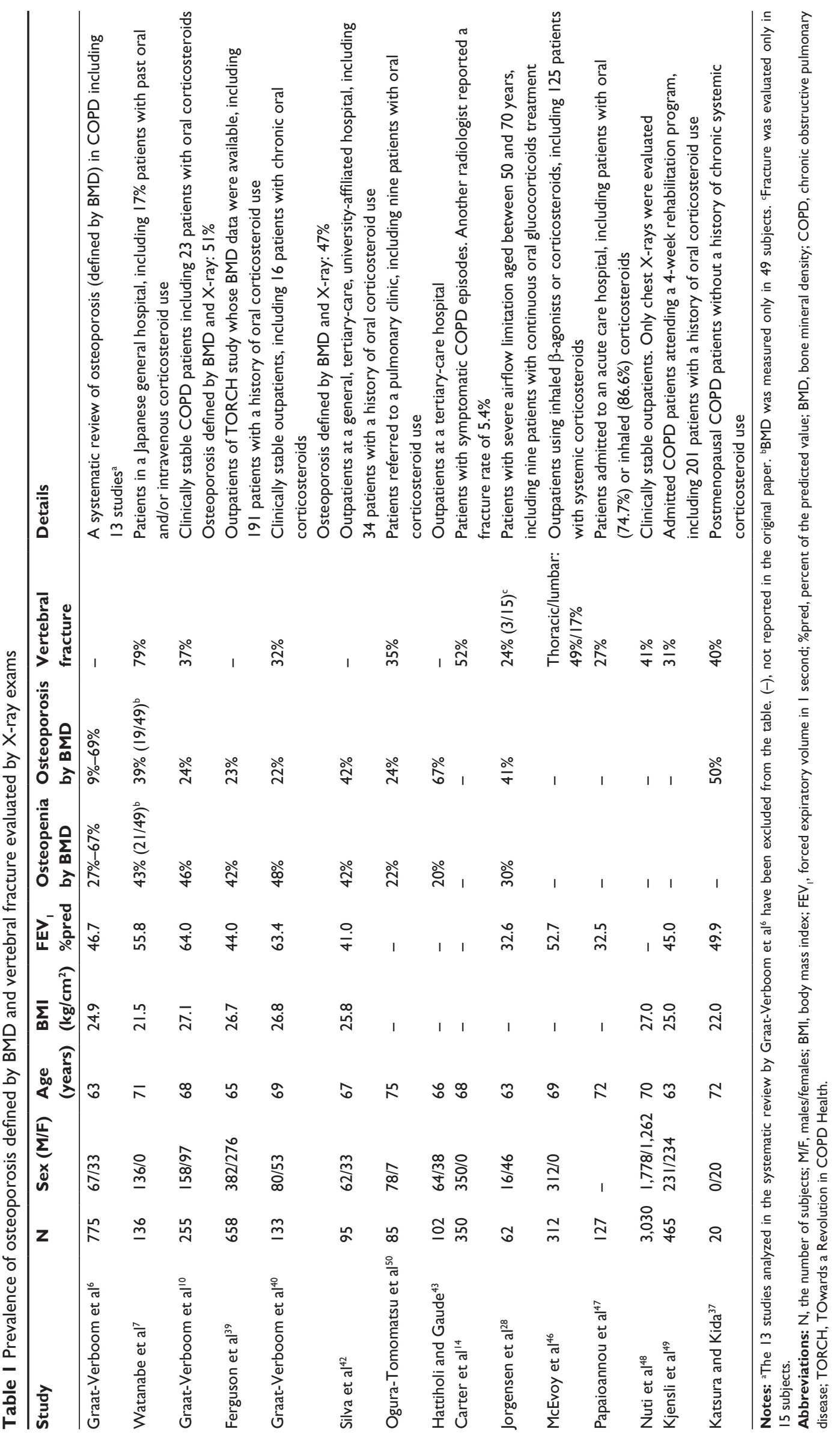


higher fracture risk in a manner independent of BMD. A large retroprospective cohort study in Manitoba investigated clinical factors associated with TBS and demonstrated that the presence of COPD was associated with lower TBS in 29,407 women aged 50 years or more, even after adjustment for age and BMD. ${ }^{59}$

Taken together, these results suggest that structural deterioration affects bone strength in COPD patients (Table 2). However, a pilot study ${ }^{60}$ comparing 30 COPD men with 17 controls using high-resolution peripheral quantitative computed tomography (CT), a powerful, noninvasive tool to assess bone microarchitecture, ${ }^{61}$ failed to demonstrate significant difference in bone structure as well as stiffness and failure load estimated by microfinite element analysis. Further studies will be necessary to establish clinical significance and contribution of bone quality to COPD-associated osteoporosis.

\section{Bone metabolism in COPD-associated osteoporosis}

Bone is subject to continuous remodeling, and the balance between resorption and formation is critical to the maintenance of bone mass and quality. Biochemical bone turnover markers are useful to noninvasively evaluate bone metabolism. ${ }^{62}$ Increase in bone markers, ie, high bone turnover, has been shown to be not only a predictor of future BMD decline but also a BMD-independent risk factor of fracture in primary osteoporosis. There are only a limited number of studies investigating bone turnover in COPD patients.

A study comparing 30 males with stable COPD with 15 age- and sex-matched ex-smoker controls found no significant differences in serum levels of P1NP or $\beta C T X{ }^{63}$ Negative results may be simply due to the small sample size, but they demonstrated that COPD patients indeed had lower BMD, which showed significant, negative correlation with the two bone markers.

However, a more recent report from China demonstrated that 50 COPD patients exhibited lower bone turnover than 50 controls: serum levels of P1NP, $\beta C T X$, and N-Mid osteocalcin were all significantly lower in COPD men than in controls. ${ }^{64}$ In COPD women, significant differences were

Table 2 Bone changes in COPD

Low BMD
Impaired bone quality
Decreased TBS
Increased cortical porosity
Low bone turnover
Suppressed bone formation

Abbreviations: BMD, bone mineral density; TBS, trabecular bone score. only observed with P1NP, suggesting that the postmenopausal status associated with high bone turnover may have masked the suppressed bone turnover in COPD. In this study, inhaled GC therapy had no significant impacts on the bone marker values.

Consistent with these results, dynamic histomorphometric analysis of biopsied bone specimens from COPD patients revealed a significantly lower trabecular mineral apposition rate compared with controls. ${ }^{57}$ It thus seems likely that COPD is associated with diminished function of bone-forming osteoblasts resulting in low bone turnover (Table 2). It should however be noted that there are multiple factors that can either enhance or suppress bone turnover to various degrees in COPD patients, including vitamin D deficiency, sex hormone deficiency, GC use, immobilization, hypoxia, and so forth. Elucidation of COPD-associated changes in bone metabolism requires further large-scale studies.

\section{Risk factors of osteoporosis in COPD}

Mechanisms by which osteoporosis occurs in COPD patients are mostly unknown. Clinical evidence has however indicated that osteoporosis and other systemic comorbidities of COPD are associated with various general and disease-specific risk factors (Table 3). 1,3,6,9,11 In the following paragraphs, we will briefly discuss general risk factors of osteoporosis present in COPD patients as well as disease-associated factors such as systemic inflammation, pulmonary dysfunction, and inhaled GC use.

\section{General risk factors for osteoporosis in COPD}

Older age and smoking are common risk factors for osteoporosis and COPD. Smoking is an established risk factor of osteoporotic fracture, but it has only a modest effect on BMD. ${ }^{65,66}$ Although the extent to which smoking contributes to fracture risk in COPD is unknown, it seems unlikely that

Table 3 Risk factors of osteoporosis in COPD

\begin{tabular}{l} 
General/common \\
Older age \\
Smoking \\
Low BMI \\
Reduced physical activity \\
Disease-specific \\
Systemic inflammation \\
Pulmonary dysfunction \\
Glucocorticoid use \\
Vitamin D insufficiency/deficiency \\
\hline
\end{tabular}

Abbreviations: COPD, chronic obstructive pulmonary disease; BMI, body mass index. 
systemic comorbidities of COPD (including osteoporosis) can be explained solely by aging and smoking. ${ }^{51,67,68}$

Body weight loss is frequently found in COPD, particularly at advanced stages, and is associated with a poor prognosis. ${ }^{69}$ In general, body mass index (BMI) is a determinant of BMD and fracture risk, and the BMI-associated fracture risk is mostly dependent on BMD. ${ }^{70}$ Consistently, low BMI has also been shown to predict osteoporosis among COPD patients. ${ }^{7,13,15}$ Cachexia in severe COPD has been attributed to systemic inflammation, with increased levels of cytokines such as TNF- $\alpha$ and increased oxidative stress. ${ }^{71}$ While both systemic inflammation and oxidative stress may cause bone metabolic abnormalities directly or indirectly through sarcopenia, how much they explain the correlation between BMD and BMI in COPD patients remains to be determined.

Mechanical loading to bone is critical to the maintenance of bone mass and integrity. Also, reduced physical activity is a known risk factor of osteoporosis in the general population. ${ }^{72,73}$ Physical activity scores have been shown to correlate with BMD in COPD patients. ${ }^{42,74} \mathrm{We}$ also found that hip BMD was negatively correlated with COPD assessment test (CAT) scores, ${ }^{7}$ which evaluate general health status and performance of COPD patients. ${ }^{75}$ Reduced physical activity may also worsen sarcopenia ${ }^{76,77}$ and reduce sunlight exposure, leading to further enhancement of bone loss.

To assess the future fracture probability, a fracture risk assessment tool called FRAX has been developed and utilized worldwide. ${ }^{78}$ FRAX calculates a 10 -year probability of fracture based on clinical risk factors including age, sex, BMI, or $\mathrm{BMD}$, a prior fragility fracture, parental history of hip fracture, current smoking, and ever use of oral GCs, and others. Of particular importance is the fact that fracture prevalence in COPD far exceeds that predicted by FRAX. ${ }^{10,50,51}$ Thus, the fracture risk posed by COPD appears to outnumber the sum of those conventional risk factors adopted in FRAX, further suggesting the involvement of unknown BMD-independent mechanisms.

\section{Systemic inflammation as a potential cause of COPD-associated osteoporosis}

The pathophysiological process of COPD is characterized by infiltration of mucosa, submucosa, and glandular tissue by inflammatory cells, resulting in increased mucous content, epithelial hyperplasia, and airway wall thickening. Chronic inflammation and imbalance between proteases and their inhibitors cause disturbed tissue repair, leading to narrowing, obliteration, and destruction of terminal bronchioles. ${ }^{3}$ Upon smoke-induced injury of epithelial cells, early cytokines such as IL-1, IL-8, and TNF- $\alpha$ are released and immune cells including macrophages, neutrophils, and dendritic cells are recruited. In addition to the basic innate immunological processes, full-blown chronic inflammatory responses involve activation of Th1, 2, 17 systems and persistent activation of resident stem cells in the lung. "Spillover" of such local inflammatory process has been hypothesized as the driver of various systemic inflammatory changes and comorbidities, including osteoporosis. ${ }^{3,79-81} \mathrm{In}$ fact, increased production of various cytokines has been demonstrated, and some of them are considered as therapeutic targets of COPD. ${ }^{81-83}$ Moreover, systemic inflammation represented by elevated CRP has been linked to osteoporosis in the general population. ${ }^{84-86}$ Consistent with a role of inflammation in COPD-associated osteoporosis, COPD patients with lower BMD have been shown to exhibit higher levels of CRP and inflammatory cytokines such as TNF- $\alpha$, IL-1, and IL-6. ${ }^{87,88}$ These three cytokines are well known inducers of osteoclasts both in vitro and in vivo and have long been considered to be involved in the pathogenesis of both primary and secondary osteoporosis, such as that associated with rheumatoid arthritis..$^{89,90}$ Consistently, it has also been reported that COPD subjects with lower BMD showed higher serum levels of RANK ligand and a higher ratio of RANK ligand/osteoprotegerin compared with those with normal BMD ${ }^{87}$ However, the spillover theory is not supported by sufficient evidence: systemic inflammation in COPD subjects is substantially heterogeneous and does not always correlate with comorbidities. ${ }^{80,91}$ And, as discussed earlier, enhanced bone resorption has not been consistently observed, arguing against a predominant role of bone resorptive cytokines in the pathogenesis of COPDassociated osteoporosis. Our preliminary results suggest that systemic inflammation in COPD is associated with impaired bone quality, evidenced by lower TBS (unpublished results by Watanabe et al). Precise roles of systemic inflammation in COPD-associated osteoporosis and its contribution to fracture risk remain to be determined.

\section{Association between pulmonary dysfunction and osteoporosis}

Both hypercapnia and hypoxia have significant impacts on bone metabolism and may predispose COPD patients to osteoporosis, ${ }^{33,92}$ particularly in patients with untreated and/or severe COPD.

Correlation between osteoporosis and pulmonary function has been relatively extensively studied. Decreased forced expiratory volume in 1 second $\left(\mathrm{FEV}_{1}\right)$ and/or advanced 
Global Initiative for Chronic Obstructive Lung Disease (GOLD) stages have been demonstrated to be correlated with lower BMD. 7,10,31,49,50 Correlation between BMD and FEV has also been demonstrated in the general population in some, but not all, reports. ${ }^{93-95}$ In COPD patients, the correlation of pulmonary function and/or GOLD stages with BMD seems to be, in part, mediated by BMI, and the correlation of GOLD stages with fracture prevalence was not always apparent. ${ }^{7,10,28}$ Although the exact reason is unclear, some mechanisms causing BMD-independent fracture risk, ie, bone quality, may be operating in COPD (as discussed earlier). In addition, GOLD stages do not always reflect time-dependent progression of COPD, and a recent study has indicated that low FEV already present in early adulthood is associated with the future development of COPD and that accelerated decline in $\mathrm{FEV}_{1}$ is not an obligate feature of COPD. ${ }^{96}$ Thus, it also seems possible that COPD subjects are already susceptible to osteoporotic fractures even at early stages due to either a particular genetic background or lower $\mathrm{FEV}_{1}$ itself.

Relationship between pulmonary function and fractures in cross-sectional studies should however be interpreted with caution, because they can mutually affect each other. Vertebral fracture can cause back pain, thoracic deformities, kyphosis, and loss of height, resulting in impaired pulmonary function. A systematic review on the relationship between pulmonary function and vertebral fractures in osteoporosis patients has demonstrated that every single vertebral fracture is associated with a $9 \%$ decline in vital capacity. ${ }^{97,98}$ Some recent studies also confirmed association of severe fractures with decreased vital capacity. ${ }^{7}$ Thus, pulmonary dysfunction could be either a result or a cause of osteoporosis, or both. One longitudinal study demonstrated that decline in $\mathrm{FEV}_{1}$ was associated with development of osteoporosis during 3 years of follow-up. ${ }^{40}$ However, current evidence is insufficient to predict development of osteoporosis from pulmonary dysfunction and vice versa.

\section{Glucocorticoid use as a fracture risk}

$\mathrm{GC}$ excess is a common and established secondary cause of osteoporosis. GC-induced osteoporosis (GIO) develops in a manner dependent on daily doses ${ }^{99}$ and occurs even at small doses. GIO is generally characterized by suppressed bone formation, ${ }^{100}$ and fracture risk in GIO is partly independent of BMD. ${ }^{20}$ Most recent studies of COPD-associated osteoporosis, however, only included a small number of subjects on systemic GCs or demonstrated increased fracture incidence in subjects without systemic GC use. ${ }^{28,47,49}$ More relevant to the fracture risk in COPD subjects is the use of inhaled GC or corticosteroid (ICS). A recent meta-analysis including 16 randomized controlled trials (RCTs) with 17,513 subjects and seven observational studies with 69,000 subjects indicated that ICS was associated with a modest but significant fracture risk $(\mathrm{OR}=1.27$ in the RCTs and 1.21 in observational studies). ${ }^{101}$ The fracture risk appeared dose-dependent. In a study of 251 COPD males, however, ICS was shown to reduce annual BMD loss in bronchitic patients, ${ }^{102}$ most likely due to ameliorated inflammation. Therefore, overall effect of ICS seems to depend on the balance between the benefit from its local anti-inflammatory effect and the fracture risk caused by its systemic effect.

\section{Vitamin D insufficiency/deficiency in COPD}

Vitamin D insufficiency/deficiency is prevalent worldwide across all ages. Vitamin D status is evaluated with serum levels of 25-hydroxyvitamin D (25D), which reflects the whole-body store of vitamin D. According to the Endocrine Society Clinical Practice Guideline, ${ }^{103}$ vitamin D deficiency and insufficiency is defined as 25D levels below 20 and 20-30 ng/mL, respectively. Vitamin D insufficiency/ deficiency leads to reduced calcium absorption from the intestine, impaired skeletal calcification, and secondary hyperparathyroidism with high bone turnover, thereby causing bone loss and increased fracture risk. It is also associated with muscle weakness and falling, which will increase fracture risk in a BMD-independent manner. ${ }^{103-105}$

Vitamin D deficiency has been shown to be highly prevalent in COPD. ${ }^{106-109}$ Moreover, 25D levels were lower in advanced stages, ${ }^{10,107-109}$ suggesting a link between COPD severity and vitamin D deficiency. ${ }^{110}$ The exact reason for such a link is unknown, although vitamin D deficiency may be caused by decreased sunlight exposure, poor diet, and smoking ${ }^{111}$ in COPD subjects. It has also been postulated that the vitamin D status may affect COPD development and exacerbations. ${ }^{112}$ Although interventions with vitamin D supplementation have not been able to provide sufficient evidence for its general efficacy, a subanalysis of subjects with $25 \mathrm{D}$ levels below $20 \mathrm{ng} / \mathrm{mL}$ demonstrated that vitamin $\mathrm{D}$ was indeed beneficial to those with vitamin D deficiency. ${ }^{113}$ A role for vitamin $\mathrm{D}$ in the long-term prognosis of COPD patients remains to be determined.

A couple of studies have demonstrated that the vitamin D status is indeed correlated with BMD in COPD subjects. ${ }^{106,107}$ One longitudinal study also demonstrated that in 100 stable COPD patients, baseline vitamin $\mathrm{D}$ deficiency increased the risk for osteoporosis development by 7.5 -fold during a 
3-year follow-up period. ${ }^{40}$ Emphasis should be put on the fact that the increase in osteoporosis during the follow-up in this study was in most cases due to newly diagnosed vertebral fracture. These results support a role for vitamin D insufficiency/deficiency in COPD-associated osteoporosis, but its contribution to the fracture risk in COPD patients should be more precisely evaluated in a larger prospective study in the future.

\section{Management of COPD-associated osteoporosis}

First of all, clinicians, particularly pulmonologists, should be aware that COPD-associated osteoporosis is extremely undertreated. Currently, there are no official guidelines for the management of COPD-associated osteoporosis..$^{1,9,11}$ Recently, a clinical five-step approach for fracture prevention in COPD was proposed based on eight clinical expert opinions in the Netherlands. ${ }^{2}$ Although neither formally approved nor validated, such a simple and stepwise approach seems easy to use in the daily practice of pulmonologists. Among these five steps (case finding, risk evaluation, differential diagnosis, therapy, and follow-up), screening procedures including case finding and risk evaluation, and therapeutic strategies appear especially important. In the following paragraphs selected topics concerning these issues will be discussed.

\section{Risk evaluation}

Any symptomatic COPD patient above 50 years of age should be screened for potential indication of pharmacological treatment (Table 4). As an initial step of risk evaluation by routine medical interviews, FRAX can be used for estimation of future fracture probability associated with general risk factors. As discussed earlier, however, FRAX underestimates the COPD-associated fracture risk. Additional risk factors such as severely impaired pulmonary function and reduced physical activity can be considered. A COPD-adapted risk score system combining FRAX and these COPD-specific

Table 4 Evaluation of fracture risk

Routine medical interview

Clinical risk factors for FRAX

Severe pulmonary dysfunction

Reduced physical activity

Height loss

Spine $X$ rays (and/or CT)

Prevalent vertebral fracture

DXA

Hip BMD

Abbreviations: FRAX, fracture assessment tools; CT, computed tomography; DXA, dual energy $X$-ray absorptiometry; BMD, bone mineral density. risk factors has been proposed. ${ }^{2}$ This system has adopted some specific risk factors that are not included in FRAX, such as immobility, more than one fall in the last year, and postbronchodilator $\mathrm{FEV}_{1}$ less than $50 \%$ predicted. ${ }^{2}$ Although these risk factors appear important in COPD-associated osteoporosis, whether or not they are truly independent of FRAX is currently unknown.

Prevalent vertebral fractures and systemic GC therapy are a strong indication of medical treatment. ${ }^{2,9}$ Lateral chest X-rays, which are routinely taken for lung evaluation, are useful to identify fractures of thoracic and upper lumbar spines. Lateral lumbar spine X-rays can also be taken as needed. The severity of vertebral fractures can be morphologically assessed according to the semiquantitative method of Genant et al. ${ }^{24}$ Chest CT images also provide information about vertebral deformities as well as bone attenuation, which has been shown to correlate with DXA-measured BMD. ${ }^{114}$ Height loss is an important piece of information that is associated with vertebral fractures. Height loss of more than $4 \mathrm{~cm}$ since the age of 25 has been shown to be associated with a 2.8-fold increase in the prevalence rate of vertebral fracture, ${ }^{115}$ and more than $2 \mathrm{~cm}$ height loss over 3 years increased new vertebral fracture risk by 13.5 -fold in postmenopausal women. ${ }^{116}$

Patients at potential risk should be examined for BMD by DXA. Hip is the standard site of BMD measurement. Although $\mathrm{BMD}$ is required for diagnosis of osteoporosis in principle, it should be noted that BMD is dispensable for FRAX.

\section{Treatment}

Due to the lack of specific evidence in COPD patients, it is recommended to basically follow general practice guidelines for the treatment of primary osteoporosis (Table 5). ${ }^{117-119}$ There may, however, be some specific considerations in COPDassociated osteoporosis, as will be discussed further. There is one RCT that specifically investigated drug efficacy in COPDassociated osteoporosis: ${ }^{120}$ One hundred forty-five subjects

Table 5 Treatment of osteoporosis in COPD

Lifestyle modifications
Smoking cessation
Exercise/physical training
Pharmacological treatment
Vitamin D and calcium
Bisphosphonate
Denosumab
Teriparatide
SERMs

Abbreviation: SERMs, selective estrogen receptor modulators. 
with airway diseases were given either placebo or alendronate, both with calcium supplements of $600 \mathrm{mg} / \mathrm{d}$, for 12 months. A significant effect was observed only in lumbar spine BMD and only with per-protocol analysis, but not with intentionto-treat analysis. So far, this is the only RCT looking at BMD changes after pharmacological interventions, and no fracture prevention studies of COPD subjects have been done.

Lifestyle modifications, including smoking cessation, ${ }^{121-123}$ healthy diet, and exercise, are beneficial to COPD patients. In particular, physical exercise has been shown to improve general conditions of COPD patients ${ }^{124,125}$ and to significantly increase BMD in postmenopausal women, ${ }^{126}$ although specific evidence that exercise improves COPD-associated osteoporosis is lacking.

As for pharmacological interventions, adequate amounts of vitamin D and calcium intake are first recommended with or without treatment with antiosteoporotic drugs. Recommended daily intake is 1,000-1,200 $\mathrm{mg}$ for calcium and 800-1,000 units for vitamin $\mathrm{D}_{3}$. Although there may be a specific repertoire of approved medications for osteoporosis in each country, first-line treatment for COPD-associated osteoporosis includes bisphosphonates such as alendronate, risedronate and zoledronate, denosumab, and teriparatide. ${ }^{117-119}$ All these drugs have been shown to be effective in the treatment of osteoporosis not only in postmenopausal women but also in men, ${ }^{119}$ and even in cases of GIO.

Oral bisphosphonates are the most commonly used drugs with considerable safety. It should however be noted that the common adverse effects of oral bisphosphonates include gastrointestinal problems. It has been demonstrated that gastroesophageal reflux disease is frequently found in COPD patients and is associated with acute exacerbations. ${ }^{127,128}$ In such cases, other bisphosphonates such as zoledronate and ibandronate can be administered intravenously. Alternatively, denosumab or teriparatide can be given subcutaneously.

Teriparatide has little evidence for hip fracture prevention but seems to be more efficacious on vertebral fractures when compared to oral bisphosphonates, particularly in men. ${ }^{129}$ Selective estrogen receptor modulators (SERMs) such as raloxifene and bazedoxifene may also be used in postmenopausal women who do not have a high risk of hip fractures, since SERMs have been shown to be effective in the prevention of vertebral fracture but not hip fracture. In some countries, including Japan, where native vitamin $\mathrm{D}_{3}$ cannot be prescribed, active vitamin $\mathrm{D}_{3}$ drugs including alfacalcidol and calcitriol may be given instead of native vitamin $\mathrm{D}_{3}$, although evidence supporting a combination therapy with antiresorptives is limited. ${ }^{130}$

\section{Conclusion}

Osteoporosis is very common in patients with COPD. BMD decline and increased fractures, particularly those of vertebrae, may involve partially independent mechanisms, which are only incompletely understood. Fractures have profound impact on the quality of life in COPD patients, but COPDassociated osteoporosis is extremely undertreated. Currently, evidence that osteoporosis treatment improves the prognosis of COPD patients is lacking. Moreover, which drugs are specifically effective in the treatment with COPD-associated osteoporosis are unknown. It seems reasonable, however, to postulate that COPD-associated osteoporosis is, at least in part, amenable to generally accepted treatment modalities. Thus, although we definitely need a specific treatment guideline for COPD-associated osteoporosis, we propose to act immediately to screen every COPD subject for osteoporosis, identify patients at high risk of fracture, and treat them with the standard medications established for primary osteoporosis.

\section{Disclosure}

Dr Daisuke Inoue reports the receipt of personal fees from Ajinomoto Pharmaceuticals and Astellas Pharma and grants from Chugai Pharmaceutical, Daiichi-Sankyo, Eli Lilly (Japan), Eisai, Pfizer, Ono Pharmaceutical, Taisho-Toyama Pharmaceutical, Takeda Pharmaceutical, and Teijin Pharma, outside the submitted work. Dr Ryo Okazaki reports receiving grants and personal fees from MSD, TEIJIN, Astellas Pharmaceuticals, Daiichi-Sankyo, Elly-Lilly, and Ono pharmaceuticals, and personal fees from Ajinomoto Pharmaceuticals and Chugai pharmaceuticals, outside the submitted work. The authors report no other conflicts of interest in this work.

\section{References}

1. Sarkar M, Bhardwaj R, Madabhavi I, Khatana J. Osteoporosis in chronic obstructive pulmonary disease. Clin Med Insights Circ Respir Pulm Med. 2015;9:5-21

2. Romme EA, Geusens P, Lems WF, et al. Fracture prevention in COPD patients; a clinical 5-step approach. Respir Res. 2015;16:32.

3. Decramer M, Janssens W, Miravitlles M. Chronic obstructive pulmonary disease. Lancet. 2012;379(9823):1341-1351.

4. Frei A, Muggensturm P, Putcha N, et al. Five comorbidities reflected the health status in patients with chronic obstructive pulmonary disease: the newly developed COMCOLD index. J Clin Epidemiol. 2014;67(8):904-911.

5. Burgel PR, Escamilla R, Perez T, et al. Impact of comorbidities on COPD-specific health-related quality of life. Respir Med. 2013;107(2) 233-241.

6. Graat-Verboom L, Wouters EF, Smeenk FW, et al. Current status of research on osteoporosis in COPD: a systematic review. Eur Respir J. 2009;34(1):209-218

7. Watanabe R, Tanaka T, Aita K, et al. Osteoporosis is highly prevalent in Japanese males with chronic obstructive pulmonary disease and is associated with deteriorated pulmonary function. J Bone Miner Metab. 2015;33(4):392-400 
8. Regan EA, Jaramillo J. It's the fracture that matters -bone disease in COPD patients. COPD. 2012;9(4):319-321.

9. Lehouck A, Boonen S, Decramer M, Janssens W. COPD, bone metabolism, and osteoporosis. Chest. 2011;139(3):648-657.

10. Graat-VerboomL, van den Borne BE, Smeenk FW, Spruit MA, Wouters EF. Osteoporosis in COPD outpatients based on bone mineral density and vertebral fractures. J Bone Miner Res. 2011;26(3):561-568.

11. Romme EA, Smeenk FW, Rutten EP, Wouters EF. Osteoporosis in chronic obstructive pulmonary disease. Expert Rev Respir Med. 2013;7(4):397-410.

12. Ryan CS, Petkov VI, Adler RA. Osteoporosis in men: the value of laboratory testing. Osteoporos Int. 2011;22(6):1845-1853.

13. Graat-Verboom L, Spruit MA, van den Borne BE, et al. Correlates of osteoporosis in chronic obstructive pulmonary disease: an underestimated systemic component. Respir Med. 2009;103(8):1143-1151.

14. Carter JD, Patel S, Sultan FL, et al. The recognition and treatment of vertebral fractures in males with chronic obstructive pulmonary disease. Respir Med. 2008;102(8):1165-1172.

15. Majumdar SR, Villa-Roel C, Lyons KJ, Rowe BH. Prevalence and predictors of vertebral fracture in patients with chronic obstructive pulmonary disease. Respir Med. 2010;104(2):260-266.

16. NIH Consensus Development Panel on Osteoporosis Prevention D, and Therapy. Osteoporosis prevention, diagnosis, and therapy. JAMA. 2001;285(6):785-795.

17. Kanis JA. Assessment of fracture risk and its application to screening for postmenopausal osteoporosis: synopsis of a WHO report. WHO Study Group. Osteoporos Int. 1994;4(6):368-381.

18. Unnanuntana A, Rebolledo BJ, Khair MM, DiCarlo EF, Lane JM. Diseases affecting bone quality: beyond osteoporosis. Clin Orthop Relat Res. 2011;469(8):2194-2206.

19. Vestergaard P. Discrepancies in bone mineral density and fracture risk in patients with type 1 and type 2 diabetes - a meta-analysis. Osteoporos Int. 2007;18(4):427-444.

20. Van Staa TP, Laan RF, Barton IP, et al. Bone density threshold and other predictors of vertebral fracture in patients receiving oral glucocorticoid therapy. Arthritis Rheum. 2003;48(11):3224-3229.

21. Lorentzon M, Cummings SR. Osteoporosis: the evolution of a diagnosis. J Intern Med. 2015;277(6):650-661.

22. Cooper C. Incidence of clinically diagnosed vertebral fractures: a population-based study in Rochester, Minnesota, 1985-1989. J Bone Miner Res. 1992;7(2):221-227.

23. Siris ES, Chen YT, Abbott TA, et al. Bone mineral density thresholds for pharmacological intervention to prevent fractures. Arch Intern Med. 2004;164(10):1108-1112.

24. Genant HK, Wu CY, van Kuijk C, Nevitt MC. Vertebral fracture assessment using a semiquantitative technique. J Bone Miner Res. 1993; 8(9):1137-1148.

25. Sattui SE, Saag KG. Fracture mortality: associations with epidemiology and osteoporosis treatment. Nat Rev Endocrinol. 2014;10(10): 592-602.

26. Bolton CE, Ionescu AA, Shiels KM, et al. Associated loss of fat-free mass and bone mineral density in chronic obstructive pulmonary disease. Am J Respir Crit Care Med. 2004;170(12):1286-1293.

27. Forli L, Mellbye OJ, Halse J, et al. Cytokines, bone turnover markers and weight change in candidates for lung transplantation. Pulm Pharmacol Ther. 2008;21(1):188-195.

28. Jorgensen NR, Schwarz P, Holme I, et al. The prevalence of osteoporosis in patients with chronic obstructive pulmonary disease: a cross sectional study. Respir Med. 2007;101(1):177-185.

29. Mineo TC, Ambrogi V, Mineo D, et al. Bone mineral density improvement after lung volume reduction surgery for severe emphysema. Chest. 2005;127(6):1960-1966.

30. Sabit R, Bolton CE, Edwards PH, et al. Arterial stiffness and osteoporosis in chronic obstructive pulmonary disease. Am J Respir Crit Care Med. 2007;175(12):1259-1265.

31. Vrieze A, de Greef MH, Wijkstra PJ, Wempe JB. Low bone mineral density in COPD patients related to worse lung function, low weight and decreased fat-free mass. Osteoporos Int. 2007;18(9):1197-1202.
32. Aris RM, Neuringer IP, Weiner MA, Egan TM, Ontjes D. Severe osteoporosis before and after lung transplantation. Chest. 1996;109(5): $1176-1183$

33. Dimai HP, Domej W, Leb G, Lau KH. Bone loss in patients with untreated chronic obstructive pulmonary disease is mediated by an increase in bone resorption associated with hypercapnia. J Bone Miner Res. 2001;16(11):2132-2141.

34. Dubois EF, Roder E, Dekhuijzen PN, Zwinderman AE, Schweitzer DH. Dual energy X-ray absorptiometry outcomes in male COPD patients after treatment with different glucocorticoid regimens. Chest. 2002; 121(5):1456-1463.

35. Incalzi RA, Caradonna P, Ranieri P, et al. Correlates of osteoporosis in chronic obstructive pulmonary disease. Respir Med. 2000;94(11): 1079-1084.

36. Karadag F, Cildag O, Yurekli Y, Gurgey O. Should COPD patients be routinely evaluated for bone mineral density? J Bone Miner Metab. 2003; 21(4):242-246.

37. Katsura H, Kida K. A comparison of bone mineral density in elderly female patients with COPD and bronchial asthma. Chest. 2002;122(6): 1949-1955.

38. Tschopp O, Boehler A, Speich R, et al. Osteoporosis before lung transplantation: association with low body mass index, but not with underlying disease. Am J Transplant. 2002;2(2):167-172.

39. Ferguson GT, Calverley PM, Anderson JA, et al. Prevalence and progression of osteoporosis in patients with COPD: results from the towards a revolution in COPD Health study. Chest. 2009;136(6): $1456-1465$.

40. Graat-Verboom L, Smeenk FW, van den Borne BE, et al. Progression of osteoporosis in patients with COPD: a 3-year follow up study. Respir Med. 2012;106(6):861-870.

41. Schnell KM, Weiss CO, Lee T, et al. The prevalence of clinicallyrelevant comorbid conditions in patients with COPD: a cross-sectional study using data from NHANES 1999-2008. BMC Pulm Med. 2012; 12(1):26.

42. Silva DR, Coelho AC, Dumke A, et al. Osteoporosis prevalence and associated factors in patients with COPD: a cross-sectional study. Respir Care. 2011;56(7):961-968.

43. Hattiholi J, Gaude GS. Prevalence and correlates of osteoporosis in chronic obstructive pulmonary disease patients in India. Lung India. 2014;31(3):221-227.

44. Dam TT, Harrison S, Fink HA, Ramsdell J, Barrett-Connor E. Bone mineral density and fractures in older men with chronic obstructive pulmonary disease or asthma. Osteoporos Int. 2010;21(8):1341-1349.

45. Chen SJ, Liao WC, Huang KH, et al. Chronic obstructive pulmonary disease and allied conditions is a strong independent risk factor for osteoporosis and pathologic fractures: a population-based cohort study. QJM. 2015;108(8):633-640.

46. McEvoy CE, Ensrud KE, Bender E, et al. Association between corticosteroid use and vertebral fractures in older men with chronic obstructive pulmonary disease. Am J Respir Crit Care Med. 1998;157(3 Pt 1): 704-709.

47. Papaioannou A, Parkinson W, Ferko N, et al. Prevalence of vertebral fractures among patients with chronic obstructive pulmonary disease in Canada. Osteoporos Int. 2003;14(11):913-917.

48. Nuti R, Siviero P, Maggi S, et al. Vertebral fractures in patients with chronic obstructive pulmonary disease: the EOLO Study. Osteoporos Int. 2009;20(6):989-998.

49. Kjensli A, Falch JA, Ryg M, et al. High prevalence of vertebral deformities in COPD patients: relationship to disease severity. Eur Respir J. 2009;33(5):1018-1024.

50. Ogura-Tomomatsu H, Asano K, Tomomatsu K, et al. Predictors of osteoporosis and vertebral fractures in patients presenting with moderate-to-severe chronic obstructive lung disease. COPD. 2012;9(4): 332-337.

51. Dennison EM, Compston JE, Flahive J, et al. Effect of co-morbidities on fracture risk: findings from the Global Longitudinal Study of Osteoporosis in Women (GLOW). Bone. 2012;50(6):1288-1293. 
52. de Vries F, Van Staa TP, Bracke MS, et al. Severity of obstructive airway disease and risk of osteoporotic fracture. Eur Respir J. 2005;25(5): 879-884.

53. Vestergaard P, Rejnmark L, Mosekilde L. Fracture risk in patients with chronic lung diseases treated with bronchodilator drugs and inhaled and oral corticosteroids. Chest. 2007;132(5):1599-1607.

54. Hippisley-Cox J, Coupland C. Derivation and validation of updated QFracture algorithm to predict risk of osteoporotic fracture in primary care in the United Kingdom: prospective open cohort study. BMJ. 2012; 344:e3427.

55. Morden NE, Sullivan SD, Bartle B, Lee TA. Skeletal health in men with chronic lung disease: rates of testing, treatment, and fractures. Osteoporos Int. 2011;22(6):1855-1862.

56. Regan EA, Radcliff TA, Henderson WG, et al. Improving hip fractures outcomes for COPD patients. COPD. 2013;10(1):11-19.

57. Kulak CA, Borba VC, Jorgetti V, et al. Skeletal microstructural abnormalities in postmenopausal women with chronic obstructive pulmonary disease. J Bone Miner Res. 2010;25(9):1931-1940.

58. Silva BC, Leslie WD, Resch H, et al. Trabecular bone score: a noninvasive analytical method based upon the DXA image. $J$ Bone Miner Res. 2014;29(3):518-530.

59. Leslie WD, Krieg MA, Hans D. Clinical factors associated with trabecular bone score. J Clin Densitom. 2013;16(3):374-379.

60. Romme EA, Rutten EP, Geusens P, et al. Bone stiffness and failure load are related with clinical parameters in men with chronic obstructive pulmonary disease. J Bone Miner Res. 2013;28(10):2186-2193.

61. Link TM. Osteoporosis imaging: state of the art and advanced imaging. Radiology. 2012;263(1):3-17.

62. Naylor K, Eastell R. Bone turnover markers: use in osteoporosis. Nat Rev Rheumatol. 2012;8(7):379-389.

63. Duckers JM, Evans BA, Fraser WD, et al. Low bone mineral density in men with chronic obstructive pulmonary disease. Respir Res. 2011; $12: 101$

64. Xiaomei W, Hang X, Lingling L, Xuejun L. Bone metabolism status and associated risk factors in elderly patients with chronic obstructive pulmonary disease (COPD). Cell Biochem Biophys. 2014;70(1):129-134.

65. Kanis JA, Johnell O, Oden A, et al. Smoking and fracture risk: a metaanalysis. Osteoporos Int. 2005;16(2):155-162.

66. Ward KD, Klesges RC. A meta-analysis of the effects of cigarette smoking on bone mineral density. Calcif Tissue Int. 2001;68(5):259-270.

67. Boyer L, Chouaid C, Bastuji-Garin S, et al. Aging-related systemic manifestations in COPD patients and cigarette smokers. PLoS One. 2015;10(3):e0121539.

68. Jaramillo JD, Wilson C, Stinson DJ, et al. Reduced bone density and vertebral fractures in smokers: men and COPD patients at increased risk. Ann Am Thorac Soc. 2015;12(5):648-656.

69. Vestbo J, Prescott E, Almdal T, et al. Body mass, fat-free body mass, and prognosis in patients with chronic obstructive pulmonary disease from a random population sample: findings from the Copenhagen City Heart Study. Am J Respir Crit Care Med. 2006;173(1):79-83.

70. De Laet C, Kanis JA, Oden A, et al. Body mass index as a predictor of fracture risk: a meta-analysis. Osteoporos Int. 2005;16(11):1330-1338.

71. Remels AH, Gosker HR, Langen RC, Schols AM. The mechanisms of cachexia underlying muscle dysfunction in COPD. J Appl Physiol (1985). 2013;114(9):1253-1262.

72. Johansson J, Nordstrom A, Nordstrom P. Objectively measured physical activity is associated with parameters of bone in 70-year-old men and women. Bone. 2015;81:72-79.

73. Nguyen TV, Center JR, Eisman JA. Osteoporosis in elderly men and women: effects of dietary calcium, physical activity, and body mass index. J Bone Miner Res. 2000;15(2):322-331.

74. Miller J, Edwards LD, Agusti A, et al. Comorbidity, systemic inflammation and outcomes in the ECLIPSE cohort. Respir Med. 2013;107(9): 1376-1384.

75. Tsuda T, Suematsu R, Kamohara K, et al. Development of the Japanese version of the COPD Assessment Test. Respir Investig. 2012;50(2): 34-39.
76. Laurent MR, Dubois V, Claessens F, et al. Muscle-bone interactions: from experimental models to the clinic? A critical update. Mol Cell Endocrinol. Epub October 23, 2015.

77. Tagliaferri C, Wittrant Y, Davicco MJ, Walrand S, Coxam V. Muscle and bone, two interconnected tissues. Ageing Res Rev. 2015;21:55-70.

78. Kanis JA, Oden A, Johansson H, et al. FRAX and its applications to clinical practice. Bone. 2009;44(5):734-743.

79. Barnes PJ. Mediators of chronic obstructive pulmonary disease. Pharmacol Rev. 2004;56(4):515-548.

80. Sinden NJ, Stockley RA. Systemic inflammation and comorbidity in COPD: a result of "overspill" of inflammatory mediators from the lungs? Review of the evidence. Thorax. 2010;65(10):930-936.

81. Hoepers AT, Menezes MM, Frode TS. Systematic review of anaemia and inflammatory markers in chronic obstructive pulmonary disease. Clin Exp Pharmacol Physiol. 2015;42(3):231-239.

82. Eagan TM, Ueland T, Wagner PD, et al. Systemic inflammatory markers in COPD: results from the Bergen COPD Cohort Study. Eur Respir J. 2010;35(3):540-548.

83. Bade G, Khan MA, Srivastava AK, et al. Serum cytokine profiling and enrichment analysis reveal the involvement of immunological and inflammatory pathways in stable patients with chronic obstructive pulmonary disease. Int J Chron Obstruct Pulmon Dis. 2014;9:759-773.

84. de Pablo P, Cooper MS, Buckley CD. Association between bone mineral density and C-reactive protein in a large population-based sample. Arthritis Rheum. 2012;64(8):2624-2631.

85. Berglundh S, Malmgren L, Luthman H, McGuigan F, Akesson K. C-reactive protein, bone loss, fracture, and mortality in elderly women: a longitudinal study in the OPRA cohort. Osteoporos Int. 2015;26(2): 727-735.

86. Ahmadi-Abhari S, Luben RN, Wareham NJ, Khaw KT. C-reactive protein and fracture risk: European prospective investigation into Cancer Norfolk Study. Bone. 2013;56(1):67-72.

87. Bai P, Sun Y, Jin J, et al. Disturbance of the OPG/RANK/RANKL pathway and systemic inflammation in COPD patients with emphysema and osteoporosis. Respir Res. 2011;12:157.

88. Liang B, Feng Y. The association of low bone mineral density with systemic inflammation in clinically stable COPD. Endocrine. 2012; 42(1):190-195.

89. Braun T, Schett G. Pathways for bone loss in inflammatory disease. Curr Osteoporos Rep. 2012;10(2):101-108.

90. Mundy GR. Osteoporosis and inflammation. Nutr Rev. 2007;65(12 Pt 2): S147-S151.

91. Barker BL, McKenna S, Mistry V, et al. Systemic and pulmonary inflammation is independent of skeletal muscle changes in patients with chronic obstructive pulmonary disease. Int J Chron Obstruct Pulmon Dis. 2014;9:975-981.

92. Arnett TR. Acidosis, hypoxia and bone. Arch Biochem Biophys. 2010;503(1):103-109.

93. Choi JW, Pai SH. Association between respiratory function and osteoporosis in pre- and postmenopausal women. Maturitas. 2004;48(3): $253-258$.

94. Jeon YK, Shin MJ, Kim WJ, et al. The relationship between pulmonary function and bone mineral density in healthy nonsmoking women: the Korean National Health and Nutrition Examination Survey (KNHANES) 2010. Osteoporos Int. 2014;25(5):1571-1576.

95. Dennison EM, Dhanwal DK, Shaheen SO, et al. Is lung function associated with bone mineral density? Results from the Hertfordshire Cohort Study. Arch Osteoporos. 2013;8(1-2):115.

96. Lange P, Celli B, Agusti A, et al. Lung-function trajectories leading to chronic obstructive pulmonary disease. $N$ Engl J Med. 2015;373(2): 111-122.

97. Harrison RA, Siminoski K, Vethanayagam D, Majumdar SR. Osteoporosis-related kyphosis and impairments in pulmonary function: a systematic review. J Bone Miner Res. 2007;22(3):447-457.

98. Leech JA, Dulberg C, Kellie S, Pattee L, Gay J. Relationship of lung function to severity of osteoporosis in women. Am Rev Respir Dis. 1990;141(1):68-71. 
99. Van Staa TP, Leufkens HG, Abenhaim L, Zhang B, Cooper C. Oral corticosteroids and fracture risk: relationship to daily and cumulative doses. Rheumatology (Oxford). 2000;39(12):1383-1389.

100. Ton FN, Gunawardene SC, Lee H, Neer RM. Effects of low-dose prednisone on bone metabolism. J Bone Miner Res. 2005;20(3):464-470.

101. Loke YK, Cavallazzi R, Singh S. Risk of fractures with inhaled corticosteroids in COPD: systematic review and meta-analysis of randomised controlled trials and observational studies. Thorax. 2011;66(8):699-708.

102. Mathioudakis AG, Amanetopoulou SG, Gialmanidis IP, et al. Impact of long-term treatment with low-dose inhaled corticosteroids on the bone mineral density of chronic obstructive pulmonary disease patients: aggravating or beneficial? Respirology. 2013;18(1):147-153.

103. Holick MF, Binkley NC, Bischoff-Ferrari HA, et al. Evaluation, treatment, and prevention of vitamin D deficiency: an endocrine society clinical practice guideline. J Clin Endocrinol Metab. 2011;96(7): 1911-1930.

104. Ebeling PR. Vitamin D and bone health: epidemiologic studies. Bonekey Rep. 2014;3:511.

105. Brincat M, Gambin J, Brincat M, Calleja-Agius J. The role of vitamin D in osteoporosis. Maturitas. 2015;80(3):329-332.

106. Forli L, Halse J, Haug E, et al. Vitamin D deficiency, bone mineral density and weight in patients with advanced pulmonary disease. J Intern Med. 2004;256(1):56-62.

107. Romme EA, Rutten EP, Smeenk FW, et al. Vitamin D status is associated with bone mineral density and functional exercise capacity in patients with chronic obstructive pulmonary disease. Ann Med. 2013;45(1):91-96.

108. Persson LJ, Aanerud M, Hiemstra PS, et al. Chronic obstructive pulmonary disease is associated with low levels of vitamin d. PLoS One. 2012;7(6):e38934.

109. Janssens W, Bouillon R, Claes B, et al. Vitamin D deficiency is highly prevalent in COPD and correlates with variants in the vitamin Dbinding gene. Thorax. 2010;65(3):215-220.

110. Zhu B, Zhu B, Xiao C, Zheng Z. Vitamin D deficiency is associated with the severity of COPD: a systematic review and meta-analysis. Int J Chron Obstruct Pulmon Dis. 2015;10:1907-1916.

111. Brot C, Jorgensen NR, Sorensen OH. The influence of smoking on vitamin D status and calcium metabolism. Eur J Clin Nutr. 1999; 53(12):920-926.

112. Janssens W, Decramer M, Mathieu C, Korf H. Vitamin D and chronic obstructive pulmonary disease: hype or reality? Lancet Respir Med. 2013;1(10):804-812.

113. Martineau AR, James WY, Hooper RL, et al. Vitamin $\mathrm{D}_{3}$ supplementation in patients with chronic obstructive pulmonary disease (ViDiCO): a multicentre, double-blind, randomised controlled trial. Lancet Respir Med. 2015;3(2):120-130.

114. Romme EA, Murchison JT, Phang KF, et al. Bone attenuation on routine chest CT correlates with bone mineral density on DXA in patients with COPD. J Bone Miner Res. 2012;27(11):2338-2343.

115. Vogt TM, Ross PD, Palermo L, et al. Vertebral fracture prevalence among women screened for the Fracture Intervention Trial and a simple clinical tool to screen for undiagnosed vertebral fractures. Fracture Intervention Trial Research Group. Mayo Clin Proc. 2000;75(9): $888-896$
116. Siminoski K, Jiang G, Adachi JD, et al. Accuracy of height loss during prospective monitoring for detection of incident vertebral fractures. Osteoporos Int. 2005;16(4):403-410.

117. Cosman F, de Beur SJ, LeBoff MS, et al. Clinician's guide to prevention and treatment of osteoporosis. Osteoporos Int. 2014;25(10): 2359-2381.

118. Kanis JA, McCloskey EV, Johansson H, et al. European guidance for the diagnosis and management of osteoporosis in postmenopausal women. Osteoporos Int. 2013;24(1):23-57.

119. Watts NB, Adler RA, Bilezikian JP, et al. Osteoporosis in men: an endocrine society clinical practice guideline. J Clin Endocrinol Metab. 2012;97(6):1802-1822.

120. Smith BJ, Laslett LL, Pile KD, et al. Randomized controlled trial of alendronate in airways disease and low bone mineral density. Chron Respir Dis. 2004;1(3):131-137.

121. Thorin MH, Wihlborg A, Akesson K, Gerdhem P. Smoking, smoking cessation, and fracture risk in elderly women followed for 10 years. Osteoporos Int. 2015;27(1):249-255.

122. Vestergaard P, Mosekilde L. Fracture risk associated with smoking: a meta-analysis. J Intern Med. 2003;254(6):572-583.

123. Gerdhem P, Obrant KJ. Effects of cigarette-smoking on bone mass as assessed by dual-energy X-ray absorptiometry and ultrasound. Osteoporos Int. 2002;13(12):932-936.

124. Reid WD, Yamabayashi C, Goodridge D, et al. Exercise prescription for hospitalized people with chronic obstructive pulmonary disease and comorbidities: a synthesis of systematic reviews. Int J Chron Obstruct Pulmon Dis. 2012;7:297-320.

125. Spruit MA, Singh SJ, Garvey C, et al. An official American thoracic society/European respiratory society statement: key concepts and advances in pulmonary rehabilitation. Am J Respir Crit Care Med.2013; 188(8):e13-e64.

126. Howe TE, Shea B, Dawson LJ, et al. Exercise for preventing and treating osteoporosis in postmenopausal women. Cochrane Database Syst Rev. 2011;(7):CD000333.

127. Kim J, Lee JH, Kim Y, et al. Association between chronic obstructive pulmonary disease and gastroesophageal reflux disease: a national cross-sectional cohort study. BMC Pulm Med. 2013;13:51.

128. Lee AL, Goldstein RS. Gastroesophageal reflux disease in COPD: links and risks. Int J Chron Obstruct Pulmon Dis. 2015;10:1935-1949.

129. Chen LX, Zhou ZR, Li YL, et al. Comparison of bone mineral density in lumbar spine and fracture rate among eight drugs in treatments of osteoporosis in men: a network meta-analysis. PLoS One. 2015;10(5):e0128032.

130. Orimo H, Nakamura T, Fukunaga M, et al. Effects of alendronate plus alfacalcidol in osteoporosis patients with a high risk of fracture: the Japanese Osteoporosis Intervention Trial (JOINT) - 02. Curr Med Res Opin. 2011;27(6):1273-1284.
International Journal of COPD

\section{Publish your work in this journal}

The International Journal of COPD is an international, peer-reviewed journal of therapeutics and pharmacology focusing on concise rapid reporting of clinical studies and reviews in COPD. Special focus is given to the pathophysiological processes underlying the disease, intervention programs, patient focused education, and self management protocols.
Dovepress

This journal is indexed on PubMed Central, MedLine and CAS. The manuscript management system is completely online and includes a very quick and fair peer-review system, which is all easy to use. Visit http://www.dovepress.com/testimonials.php to read real quotes from published authors. 